\title{
Ocio y homosexualidad: un estudio etnográfico sobre el asociativismo deportivo de mujeres, en el contexto de un deporte dicho masculino
}

\author{
Marco Paulo Stigger* \\ Raquel da Silveira**
}

\begin{abstract}
Resumen: En este estudio se intenta comprender el asociativismo deportivo de mujeres, en el contexto de un deporte dicho masculino; se trata de una investigación etnográfica junto a un equipo de futsal femenino de la ciudad de Porto Alegre, en Brasil. Conducidos por el objetivo de comprender cómo y por qué algunas mujeres se asocian para practicar un deporte socialmente considerado como masculino, constatamos que el asociativismo estudiado se vincula al placer de la práctica deportiva y a la homosexualidad. Identificamos una heterogeneidad en lo que se refiere a la forma de vivir el deporte y nos percatamos de que, en ese universo, la homosexualidad es vivida, pero se le da una visibilidad controlada; observamos, también, que el contexto deportivo estudiado se constituye en un espacio de ocio particular, donde la homosexualidad se acepta, siendo, por lo tanto, un espacio privilegiado para que las mujeres homosexuales vivan sus momentos de ocio.
\end{abstract}

Palabras clave: Ocio, homosexualidad, futsal, deporte

\section{Leisure and homosexuality: an ethnographic study on sport associativism of women, in the context of a so-called male sport}

\begin{abstract}
This study seeks to understand the sport associativism of women in the context of a so-called male sport. It is an ethnographic research on a female indoor soccer team from the city of Porto Alegre, Brazil. Aiming to understand how and why women associate to practice a sport socially considered masculine, we acknowledged that the studied associativism is connected to the pleasure of the practice and to homosexuality. We identified an heterogeneity on the way of living the sport and realized that, in that universe, homosexuality is lived, but its visibility is administered; we also observed that the studied sportive context is constituted of a space of private leisure where homosexuality is accepted, making it a privileged space for homosexual women to spend their moments of leisure.
\end{abstract}

Key words: leisure, homosexuality, futsal, sport

\footnotetext{
* Universidade Federal do Rio Grande do Sul (UFRGS), Porto Alegre, Brasil, stigger@adufrgs.ufrgs.br

** Universidade Federal do Rio Grande (FURG), Rio Grande, Brasil, raqkarate@hotmail.com
} 


\section{Lazer e homossexualidade: um estudo etnográfico sobre 0 associativismo esportivo de mulheres, no contexto de um esporte dito masculino.}

Resumo: Este estudo visa compreender o associativismo esportivo de mulheres, no contexto de um esporte dito masculino; trata-se de uma pesquisa etnográfica junto a uma equipe feminina de futsal na cidade de Porto Alegre, Brasil. Conduzidos pelo objetivo de compreender como e porque algumas mulheres se associam para praticar um esporte socialmente considerado como masculino, constatamos que o associativismo estudado está ligado ao prazer da prática esportiva e à homossexualidade. Identificamos uma heterogeneidade no que diz respeito à forma de viver o esporte e percebemos que, neste universo, a homossexualidade é vivida, mas tem uma visibilidade controlada; observamos também que o contexto esportivo estudado se constitui em um espaço de lazer particular, onde a homossexualidade é aceita, sendo, portanto, um espaço privilegiado para que as mulheres homossexuais vivam seus momentos de lazer.

Palavras-chave: Lazer, homossexualidade, futsal, esporte

Recibido: 11.05.2010

Aceptado: 30.06.2010

$$
* * *
$$

\section{Introducción}

en los siglos XVIII y XIX, el deporte y la gimnasia fueron inventados

por el hombre y para el hombre, ellos desarrollaron las actividades, prácticas y actuaciones deportivas de acuerdo con sus propias necesidades e ideales. (...) Hoy, el deporte aún es un mundo masculino, pero las

mujeres están haciendo sentir su presencia

Pfister, 2003:11.

No es difícil estar de acuerdo con Pfister, cuando, en la afirmación anterior, llama la atención sobre el protagonismo masculino en el mundo de los deportes. Y, si la afirmación es verdadera en el contexto general de los deportes, mucho más evidente se mostrará si el foco son los deportes considerados socialmente como masculinos, lo que es el caso del fútbol, en Brasil. Esta evidencia, que se muestra clara en la realidad social brasileña, también se refleja en el contexto de las prácticas de entretenimiento y ocio de mujeres. Difícilmente encontramos mujeres jugando fútbol en los fines de semana, al contrario de lo que ocurre con los hombres que, según observaciones del cotidiano brasileño y lo que ha sido observado en algunos estudios, lo tienen como una de las prácticas preferidas de fin de semana. En Brasil son muy pocas las investigaciones sobre la presencia de las mujeres en el fútbol y sus derivados ${ }^{1}$ practicados en los momentos de ocio, en

\footnotetext{
${ }^{1}$ Denominamos derivaciones del fútbol aquellas prácticas deportivas que se aproximan del fútbol, pero son desarrolladas, en Brasil, con diferentes características y denominaciones.
} 
comparación a la producción académica destinada a esa discusión sobre el fútbol practicado por los hombres.

Algunos ejemplos de estudios académicos sobre ocio pueden ser ofrecidos para ilustrar cómo los hombres -más que las mujeres- optan por prácticas deportivas en su tiempo de ocio (Soares, 1994; Soares y Lovisolo, 2001; Toledo, 2002; Damo, 2002; Betti, 2004; Moura, 2005; Daolio, 2005; Damo, 2006; Guedes, 2006). Pero como también señala Pfister, es igualmente verdad que ese dominio masculino en el mundo deportivo no significa que las mujeres no sean parte de ese universo. Aunque de modo reducido, si se compara con la participación de los hombres en los deportes, en los días de hoy es evidente que ellas, efectivamente, están presentes en el mundo deportivo, incluso cuando este mundo forma parte del ocio de las mujeres. Así, aunque en un número muy inferior a los estudios en que los hombres son los protagonistas, actualmente ya existen trabajos que reflejan el interés académico sobre la presencia femenina en los deportes practicados en los momentos de ocio, incluso en lo que se refiere a los deportes dichos y/o considerados masculinos.

Este es el caso del fútbol, un deporte tradicionalmente visto como masculino en el contexto brasileño (Moura, 2005), pero que hoy, siendo practicado por mujeres, está despertando significativo interés por parte de los intelectuales. Después de analizar la forma en que los estudios sobre la participación de las mujeres en el universo del fútbol se expresan en la realidad académica brasileña, identificamos que en la mayor parte de esos trabajos las mujeres quedan en un segundo plano: aunque las mujeres estén presentes como foco de varias investigaciones, los hombres son los que constan como quienes practican el fútbol. Un ejemplo de eso es el trabajo de Costa (2007), que parte de la pregunta: “¿qué es una "hincha” de fútbol?”. Pero también es posible encontrar estudios con otro abordaje, un abordaje en que la atención se dirige a las mujeres, en situaciones en que éstas son las protagonistas (practicantes) del fútbol (Dornelles, 2004).

Así, aunque existan algunos estudios sobre las mujeres y sus relaciones con deportes considerados masculinos en los momentos de ocio, en la mayoría de ellos éstas ocupan un lugar secundario; por esta razón, en el ámbito académico poco se sabe sobre cómo ocurren esas prácticas cuando son realizadas por las mujeres. Estimulados por esta constatación, desarrollamos una investigación con el objetivo abarcar aspectos de un deporte que es socialmente visto como masculino cuando éste es practicado por mujeres en su tiempo de ocio. Para eso, optamos por tomar, como universo empírico de la investigación, a un grupo de mujeres practicantes de

Damo (2003) agrupa la diversidad futbolística en configuraciones denominadas: fútbol profesional, fútbol de bricolage, fútbol comunitario y fútbol escolar. Además de estas, nos referimos a aquella practicada por el grupo de mujeres estudiado en este trabajo: el Futsal, una modalidad que, así como el fútbol, en Brasil es socialmente considerada como un deporte masculino. 
futsal ${ }^{2}$, en la ciudad de Porto Alegre, en Brasil. En ese contexto, preguntamos: ¿cómo y por qué mujeres practican un deporte socialmente considerado masculino en sus momentos de ocio? ¿Cuáles son las estrategias y formas de funcionamiento adoptadas por el grupo? ¿Qué significados atribuyen al fútbol que practican? ¿Cómo se incorpora a sus vidas la práctica del fútbol?

La búsqueda de respuestas para esas interrogantes nos llevó a la comprensión de varios aspectos de la vida deportiva de esas mujeres, entre los cuales el hecho de que entre ellas había un gran número de mujeres homosexuales. Eso nos permitió darnos cuenta de que sus actividades de entretenimiento vinculadas al universo deportivo del cual forman parte se constituyen en un espacio de sociabilidad homosexual. Además de otros aspectos que serán tratados, es con ese foco que presentaremos los resultados de la investigación realizada. Esa investigación se caracterizó como un estudio etnográfico desarrollado en 12 meses de trabajo de campo. En un primer momento, delinearemos los aspectos metodológicos que condujeron la investigación y después presentaremos los resultados en sí.

\section{Etnografía: una mirada desde adentro}

La perspectiva de centrar la atención en una expresión particular del deporte, aquella practicada en los momentos de ocio, nos llevó a desarrollar una investigación etnográfica, una forma de mirar hacia los fenómenos culturales anclada en la tradición antropológica de investigación ${ }^{3}$. Surge de los esfuerzos de los pueblos europeos, cuando intentaban comprender las costumbres extrañas de las poblaciones con las que entraban en contacto en sus viajes por otras regiones del planeta. Esta especie de ciencia de la traducción pasó a reconocer que los comportamientos colectivos no son naturales, sino construidos socialmente y, por más extraños que parezcan, tienen sentido para los integrantes de los respectivos grupos sociales. Sin embargo, si esta tradición surge de la voluntad de transformar lo extraño en familiar, al intentar también entender aspectos culturales de su propia sociedad los antropólogos pasaron a seguir un camino inverso: trataron de transformar lo familiar en extraño. En este camino, empezaron a identificar que, en el contexto heterogéneo de los grandes núcleos urbanos, hábitos y valores que parecen ser muy similares se muestran, a veces, bastante diferentes (Magnani, 1984; Damatta, 1987; Laplatine, 1994).

\footnotetext{
${ }^{2}$ Como ya mencionamos anteriormente, consideramos el Futsal como un derivado del fútbol, tanto por sus características funcionales, como por el hecho de que también es un deporte, en Brasil, considerado masculino. Por estas razones, y también por el hecho de que las mismas mujeres también practican el fútbol, a partir de aquí trataremos igualmente el futsal y el fútbol.

${ }^{3}$ Howe (2001: 293), al referir que la etnografía se ha constituido en una importante perspectiva para los estudios sobre el deporte, afirma que la Antropología es la "tradicional "casa” de la etnografía".
} 
Es en este universo en que se insierta el estudio ahora presentado, que tuvo la intención de comprender las peculiaridades de un deporte (dicho) masculino, cuando es practicado por mujeres en los momentos de ocio. El análisis fue realizado desde adentro, dando prioridad a la experiencia personal en campo, lo que es una característica fundamental de los estudios etnográficos, vistos como emprendimientos intelectuales que buscan desarrollar descripciones densas de los contextos culturales investigados (Geertz, 1989).

La comprensión de estos significados se buscó a partir de la observación participante, la cual consiste -en líneas generales- en la vivencia del investigador, por un largo periodo, en el contexto que pretende investigar. En esta experiencia, el investigador -un extranjero en el universo cultural en que está ahora implicado- estará en contacto con modos de vida donde están presentes diferentes sistemas de significación, valores y comportamientos sociales que pretende desvelar. Así, desarrollará su investigación a través de la participación continua en la vida de estos sistemas culturales particulares, y, en base a los datos obtenidos por la observación directa y sistemática, intentará interpretarlos en su globalidad (Damatta, 1987; Becker 1997). Entre los aspectos fundamentales del estudio etnográfico están el mirar, el escuchar y el escribir (Oliveira, 1998) y/o el "arte de ver, arte de ser, arte de escribir” (Winkin, 1998, p. 132). Relacionando las propuestas de estos autores y especificando para trabajos etnográficos relacionados a los deportes, Stigger (2007) considera necesario aprender a convivir deportivamente con los grupos investigados.

El trabajo de campo se desarrolló de agosto del 2006 a agosto del 2007, cuando realizamos 51 visitas a campo, principalmente en los encuentros semanales del grupo investigado, que ocurrían los sábados (29) y los domingos (10). También tuvimos la vivencia de otros momentos del cotidiano de las integrantes del grupo, como fiestas de cumpleaños, almuerzos y cenas, además de otros momentos aleatorios.

También fueron realizadas 17 entrevistas con participantes del equipo. Entendiendo que las entrevistas son "un escuchar muy especial" (Oliveira, 1998: 22) y que deberían obedecer al "principio de diversificación de las personas” (Ruquoy, 1997: 103), éstas fueron realizadas dos meses después de terminada la observación participante -esa estrategia posibilitó escoger a los informantes que consideramos más adecuados al interés del estudio, permitiendo abarcar diferentes aspectos de la realidad observada. Las entrevistas fueron transcritas y devueltas a las personas entrevistadas; tras este proceso de validación, cada informante autorizó el uso del material obtenido, mediante firma de un Término de Consentimiento Libre y Esclarecido.

Otra fuente de informaciones fue la utilización de la internet: autorizados por el grupo, tuvimos acceso a cerca de 150 mensajes que circulaban entre las jugadoras y también a las conversaciones trabadas por la comunidad creada por el grupo (Orkut). 


\section{“A mí lo que me gusta es jugar!”: el equipo de futsal y las formas de apropiación del fútbol en los momentos de ocio}

El Equipo 4 investigado está compuesto por 17 practicantes amadoras de fútbol, que tienen entre 16 y 41 años de edad. Algunas viven en Porto Alegre, otras en ciudades cercanas. La mayoría de ellas tiene una vida profesional activa y las profesiones son bastante diversificadas; están, además, aquellas que estudian. Forman parte del equipo desde personas con formación y actuación profesional de nivel universitario (administradoras de empresas; fisioterapeuta; química) hasta estudiantes de enseñanza básica y personas que actúan en profesiones no especializadas (empleada doméstica; office girl).

El surgimiento del equipo fue producto de la iniciativa de dos de las jugadoras, pero fue con la ayuda de Pedro (profesor de educación física y técnico voluntario) que Julia y Laura se iniciaron en actividades más sistemáticas. Además de éstos y de las demás jugadoras, también son consideradas como parte del grupo personas a él vinculadas de distintas formas, pero siempre a partir de algún tipo de vínculo con las participantes. Son amigos, amigas, novios, novias, maridos, compañeras ${ }^{5}$, hijos y sobrinos que están sistemáticamente presentes en diversas actividades.

Interrogadas sobre qué es lo que más las aproxima, unánimemente responden que la práctica del futsal es el vínculo más fuerte entre la mayoría de las integrantes; sobre ese aspecto, llama la atención que a pocas de ellas les guste asistir al fútbol, sea en los estadios o en la televisión. Como afirma Graciele, lo que les gusta “es jugar”.

Queda claro, también, que sus trayectorias deportivas en lo que se refiere al fútbol son semejantes. Varias de ellas declararon que ya jugaban fútbol desde la infancia, cuando practicaban en la calle y entre los chicos, lo que se coaduna con otros estudios realizados en Brasil. En algunos de esos trabajos se hace evidente que, en Brasil, el fútbol es un universo de socialización masculino, donde la presencia de grupos de niñas practicando el fútbol en la calle termina siendo una excepción a la regla. Pese a no haber sido foco de los estudios mencionados anteriormente, tanto Damo (2007) como Thomassim (2007) se dieron cuenta de la presencia de chicas iniciándose en ese deporte junto a grupos de chicos. Sin embargo, comparando los resultados de esos dos trabajos fue posible observar diferencias en lo que se refiere a la forma en que los chicos y las chicas se identificaron al practicar el fútbol, cuando lo hacen juntos. El estudio de Damo (2007) -realizado entre jóvenes de clase media y en un barrio cercano al centro de la ciudad- mostró una gran resistencia de los chicos en relación a la presen-

\footnotetext{
${ }^{4}$ Para no exponer el nombre del equipo investigado, nos referiremos a él como "Equipo", con la primera letra en mayúscula.

${ }^{5}$ Término nativo que expresa la idea de una convivencia conyugal (o casi conyugal), aunque sin vínculo legal. Entre éstos se incluyen los novios y las novias.
} 
cia de una chica en sus encuentros deportivos; y la misma resistencia ocurría por parte de los padres en cuanto al hecho de que a su hija le gustara jugar fútbol. Thomassim (2007), a su vez, en estudio desarrollado en la periferia de la ciudad y entre niños pobres, observó que la presencia de niñas entre los niños era algo "invisible" (p. 105); en ese estudio, preguntado sobre cómo veía la presencia de una chica en su equipo, uno de los chicos (Duda) contestó: “¿qué chica?”.

Pero a pesar de haberse iniciado junto a los chicos, actualmente ninguna de las practicantes investigadas juega sistemáticamente con hombres. Cuando decimos que ellas no lo hacen sistemáticamente, es porque algunas veces se incorporan a juegos de fútbol de grupos masculinos, lo que ocurre sólo cuando las relaciones personales son bastante cercanas. Fue lo que dijo Valéria, cuando afirmó que a veces la invitan para que complete el equipo de su hermano y/o de amigos cuando faltan jugadores en sus encuentros deportivos; eso también ocurre en sus entrenamientos, pero de modo inverso: si faltaban jugadoras, se llamaba a los novios para conformar los equipos.

Pero aunque actualmente las practicantes investigadas jueguen sistemáticamente sólo con mujeres, el fútbol practicado por ellos sigue presente de manera significativa en los discursos, comparaciones y en los gestos de ellas. En diversos momentos, observamos que las practicantes de futsal analizaban las jugadas unas de las otras a partir de lo que consideraban un buen fútbol, es decir, el fútbol masculino. Al enfatizar acciones deportivas consideradas bastante positivas, la comparación siempre se hacía con relación al fútbol de los hombres: “ella se tira como si fuera arquero en serio”6 dijo Graciele (17/9/2007), refiriéndose a cómo los arqueros (hombres) actúan. En Brasil, este tipo de comparación también se da en los medios de comunicación: siempre que la Selección Brasileña Femenina de Fútbol presenta buenos resultados, se hace un paralelo entre el grupo (y cada jugadora en particular) y la Selección Masculina de Fútbol. Decir que alguna de ellas es la "Ronaldinha"7 es un tipo de comparación recurrente.

\section{Entrenar, jugar y convivir: momentos de una práctica deportiva particular}

Con esas características, las participantes del Equipo incorporan sus prácticas entre sus actividades de ocio, ya que las viven en momentos de no trabajo y son destinadas a su interés propio (Dumazedier 1973; Elias \& Dunning, 1992). Son actividades que forman parte del universo del fútbol amador de la ciudad, y que, pese a que la participación es voluntaria, atraen a un conjunto significativo de practicantes ${ }^{8}$. Sin embargo, a pesar de la

\footnotetext{
${ }^{6}$ Destaque nuestro.

${ }^{7}$ Referencia al atleta "Ronaldinho”, más de una vez considerado como el mejor jugador de fútbol del mundo.

${ }^{8}$ En uno de los campeonatos de que nuestras colaboradoras participaron, habían 20 equipos inscritos, en un total aproximado de 200 mujeres.
} 
libertad de elección en cuanto a participar o no en el grupo, una vez que están en él, ellas pasan a asumir compromisos tanto en los entrenamientos que semanalmente son desarrollados como en los juegos de los que participan en torneos y campeonatos. Con ese espíritu, estrategias y jerarquías fueron siempre visibles, desde el momento mismo en que alguna jugadora era candidata a incorporarse al Equipo. Para que la acepten en el grupo, la potencial integrante necesita pasar por un test capaz de mostrar que tiene “capital deportivo” (Marivoet, 1998: 31) compatible con el equipo. El modo de ingreso, por lo tanto, es una indicación de que la actuación/rendimiento permea la manera en que el Equipo vive el deporte en el ocio.

La admisión también debe ocurrir a través de invitación/indicación de las candidatas, donde otras cualidades son evaluadas. Durante el periodo en que observamos al grupo, eso ocurrió tanto por invitaciones de amigas como a partir de las cualidades deportivas de alguna jugadora, observada por Pedro (el técnico) en juegos y campeonatos; en ese caso, Pedro invitaba a la jugadora a que se presentara a un entrenamiento, donde era evaluada. Después de una primera aceptación, además de demostrar sus atributos deportivos la nueva integrante tiene que asumir un conjunto de compromisos, como estar presente en días y horarios acordados y dedicarse con seriedad al grupo, ya que, en ese universo, no presentarse al entrenamiento y/o al juego sin avisar/justificar se considera como una falta grave. Ana, al explicar por qué no invitar a algunas buenas jugadoras para que participen en el Equipo afirma: "no, porque ellas no tienen muchas ganas de entrenar”.

Aunque entrenamientos y juegos están articulados entre sí y son vividos por las mismas personas, la convivencia cotidiana permitió percatarnos de algunas peculiaridades en lo que se refiere a las diferentes formas con que el fútbol es vivenciado por ellas. Aquello que las informantes denominaban "entrenamientos" se desarrollaba siempre en un mismo gimnasio de deportes, donde había una cuadra que era alquilada por dos horas semanales, los sábados por la tarde. Los juegos oficiales se realizaban en diversos gimnasios de la ciudad y sus alrededores, casi siempre los domingos.

Observando los entrenamientos, nos dimos cuenta de que, la mayoría de las veces, la capacidad de producir deportivamente era importante. En esos momentos, Pedro investía en el desarrollo de las técnicas de los movimientos y en la organización táctica del equipo para los juegos que éste disputaría; de la misma forma, las jugadoras se esforzaban para perfeccionar esos fundamentos del futsal y también para demostrar sus habilidades a las demás y al técnico, en la perspectiva de colocarse en la condición de titular o reserva del Equipo. Pero también fue posible encontrar diferencias entre los partidos/entrenamientos que se desarrollaban en el ámbito interno ${ }^{9}$ y los que ocurrían contra equipos externos ${ }^{10}$. Si en la se-

\footnotetext{
${ }^{9}$ Apenas entre componentes del Equipo.

${ }^{10}$ Ocasionalmente, otros equipos eran invitados a participar de los entrenamientos, realizando "partidos amistosos".
} 
gunda situación el nivel de seriedad afloraba y todas las jugadoras intentaban mostrar "su juego", en la primera la actividad se caracterizaba por ser un momento de diversión, donde un clima de juegos y chanzas llamaba la atención.

Los partidos oficiales ${ }^{11}$ son aquellos realizados en torneos y en campeonatos que ocurren en la ciudad, organizados por grupos de practicantes y/o por las Municipalidades de Porto Alegre y sus alrededores. Los torneos son competiciones que ocurren en uno o dos días, y se desarrollan en mañana, tarde y noche en un solo gimnasio. Los campeonatos son competiciones que duran de tres a cuatro meses, cuyos partidos son realizados en días y horarios diversos.

Durante esos partidos, es visible el esfuerzo de las jugadoras para tener una buena actuación y -con mucha frecuencia- entre ellas hay mucha tensión. Si en algunas situaciones las victorias son muy celebradas, en otras las derrotas llegan a causar disputas entre las jugadoras, el técnico y la hinchada del Equipo. Esa tensión se muestra más evidente cuando la derrota y/o victoria ocurren sobre algún equipo que, en la historia de sus encuentros deportivos, se ha venido configurando como un equipo rival. En esos momentos es cuando se puede percibir, de manera más clara, las expectativas establecidas entre cada una de las participantes y entre ellas y el grupo. Es en las competiciones que se hace evidente quién, efectivamente, pertenece al grupo, aspecto que toma en cuenta tanto el asumir compromisos con el grupo como la capacidad de contribuir para las victorias del Equipo. Sin embargo, además de esas exigencias ya mencionadas, no fueron pocos los testimonios que llamaron la atención hacia otras formas de sociabilidad entre las jugadoras. Aunque, la mayor parte del tiempo, la seriedad y la búsqueda por el resultado deportivo sea perceptible, eso no impide que, en otros, la informalidad y la diversión salten más a la vista. Si el rendimiento deportivo es un factor que excluye a algunas jugadoras del Equipo, eso no es lo único que determina el funcionamiento del grupo. Además de jugar bien y asumir compromisos colectivos que favorezcan los resultados en los juegos, también se valoriza el hecho de compartir códigos peculiares del equipo, como participar en fiestas, asados y otras actividades de ocio; también se espera alguna participación en aspectos de la vida personal unas de las otras. Son ejemplos de esas situaciones los diversos asados en que participamos, los varios encuentros para jugar paddle con algunas de las participantes del grupo, así como la presencia de casi todas las integrantes en la inauguración del restaurante de Laura y Rossana ${ }^{12}$.

\footnotetext{
${ }^{11}$ En Porto Alegre, ninguna entidad vinculada a las organizaciones oficiales del Futebol Brasileño es responsable por las competiciones a que nos referimos. Cuando las caracterizamos como oficiales, es en el sentido de que - pese a que tienen un carácter de informalidad - forman parte de un evento bastante reconocido por el universo nativo.

${ }^{12}$ Laura fue una de las personas que creó el equipo y actualmente juega en él. Rossana es la compañera de Laura y, a pesar de no jugar fútbol, acompaña el equipo desde los entrenamientos hasta las competiciones.
} 
La exigencia de una buena actuación deportiva, sumada a la necesidad de asumir diferentes tipos de compromisos con el grupo, también fue encontrada por Stigger (1997: 53) en estudio sobre el fútbol practicado en el contexto de los "Veteranos del Ararigbóia"13. En aquel contexto,

la presencia durante todo el periodo [era] un factor importante que [establecía] la diferencia entre quien efectivamente [participaba] del grupo y quien "solamente [venía] a jugar fútbol”: participar del almuerzo [era] fundamental, pues muchas veces "el equipo se configura en el asado”.

Así, aunque las actividades por ellas desarrolladas estuviesen incorporadas al tiempo libre, también se caracterizaban por una práctica de ocio con significativo nivel de seriedad, es decir, con "un tipo de dedicación laboriosa” (Rybczynski, 1991: 38), donde es necesario "hacer bien las cosas” (Rybczynski, 2000: 187), una exigencia jerarquizaba a las participantes a partir de lo que fuesen capaces de producir. Por otro lado, en especial en las actividades internas del grupo, parece que esas mujeres también estaban unidas por un tipo de sociabilidad vivida como una forma autónoma o lúdica de asociación, un juego simbólico libre de lazos de contenido, donde algunas diferencias sociales son reelaboradas (Simmel, 1983).

En las páginas siguientes, tendremos la oportunidad de reflexionar sobre otras características del grupo estudiado, las cuales guardan relación con el hecho de que ellas son practicantes de un deporte que, en Brasil, es reconocido como masculino. A diferencia de lo que usualmente se encuentra en contextos de fútbol masculino, en los que la heterosexualidad es reforzada, entre las mujeres estudiadas fue tan grande el número de bisexuales y homosexuales que llamó nuestra atención. Además de las cuestiones que el tema deporte y género suscitó, presentaremos algunos aspectos vinculados al manejo de la homosexualidad en el contexto del grupo y en las relaciones del Equipo con su exterior.

\section{El manejo de la visibilidad de la homosexualidad en el universo del futsal}

El tema de la sexualidad está presente en muchos estudios que abordan los deportes socialmente considerados masculinos cuando son practicados por mujeres (Mennesson 2004, 2005; Mennesson y Clément 2003; Dornelles 2004; Knoppers y Anthonissen, 2003). El tema llama la atención, tanto por ser una oportunidad para debates sobre la diversidad de formas de vivir la sexualidad como por el hecho de que - en esos contextos muchas veces son encontradas mujeres que rompen con la "matriz hetero-

\footnotetext{
${ }^{13}$ En el universo estudiado, eran considerados "veteranos" los hombres con edad superior a 40 años que participaban de un grupo que practicaba el fútbol en un parque público de la ciudad, denominado Parque Ararigbóia.
} 
sexual [que] delimita los estándares que deben ser seguidos” (Louro, 2004: 17). En el Equipo eso no ocurre en forma diferente. En el universo que estudiamos fueron encontradas mujeres heterosexuales, pero también bisexuales y homosexuales. Fue el número significativo de mujeres bisexuales y homosexuales identificadas lo que nos llevó a considerar importante reflexionar sobre esa característica del grupo y preguntar por qué y de qué manera esas mujeres estaban presentes en ese universo deportivo.

En la búsqueda por esas respuestas, seguimos las enseñanzas de Touraine (2007: 9) y decidimos "visitar el campo y, sobre todo, en vez de hablar en nombre de ellas, escucharlas”. La propuesta fue en el sentido de comprender cómo se entrelazaba la sexualidad entre las practicantes del futsal a partir de lo que vivenciamos durante la investigación. Con esa perspectiva, nuestra intención no fue la de generalizar los hallazgos del trabajo, sino identificar cómo nuestras colaboradoras vivenciaban la homosexualidad femenina.

En ese intento de expresar lo que presenciamos en el transcurso de la investigación, la experiencia de campo nos llevó al manejo de la visibilidad de la homosexualidad, tanto en el medio futbolístico como fuera de ese medio, siendo este el aspecto que condujo el análisis. Esa categoría nos ayudó a comprender, a partir de sus ramificaciones, la manera en que las jugadoras investigadas viven la homosexualidad en el futsal e, incluso, fuera de él.

Hablar de visibilidad e invisibilidad de la homosexualidad tiene sentido debido al hecho de que la sexualidad está estrechamente ligada a la norma "pareja [heterosexual], legítima y procreadora” (Foucault, 2006: 9), lo que hace con que existan muchas mujeres que "manejan la visibilidad de sus relaciones homoeróticas” (Meinerz, 2005: 122). En el grupo que investigamos, esa posibilidad se confirmó: desde que iniciamos la investigación con las practicantes de futsal, presenciamos la existencia de mujeres que están fuera del patrón heterosexual, así como muchas estrategias que algunas de las participantes del grupo utilizan para manejar la visibilidad de su homosexualidad; también identificamos preocupaciones con el manejo de la imagen del grupo, en la relación con los demás grupos de practicantes de futsal femenino.

En entrevista, Laura deja claro que saber manejar la visibilidad de su sexualidad es algo que aprendió "viviendo (...), con el tiempo" (Laura, 30/10/2007). Refiriéndose a los prejuicios de que ya fue víctima e intentando llamar la atención sobre que su vida no se resume a su sexualidad, enfatizó:

yo no soy "la homosexual Laura", yo soy "Laura, tam, tam, tam, tam, homosexual”, ¿entiendes? Entonces, eso no es mi principal. Eso forma parte de mi vida, pero no es mi todo. Y yo creo que cuando tú planteas eso, parece que ya creas una barrera (Laura, 30/10/ 2007). 
Inicialmente Laura está diciendo que su existencia no se centra en su vida sexual, pues al mismo tiempo en que es homosexual tiene una vida profesional, familiar, juega futsal y le gustan muchas otras actividades que no pueden ser vinculadas a su sexualidad. Definir a Laura a partir de la homosexualidad sería, por lo tanto, reducir su vida a una esfera que, según ella, "no es mi todo". Esa idea se acerca a lo que afirma Touraine (2007: 68), cuando defiende que

No existe ninguna razón para pensar que los gays o las lesbianas tienen conductas específicas. La forma de prejuicios contra los o las homosexuales, gays y lesbianas, más difícil de destruir es la creación de imágenes globales, como si la homosexualidad definiese un modelo absoluto de personalidad y de acción, idea que nadie osa aplicar a los heterosexuales.

Pero Laura también se refiere a formas explícitas e implícitas de prejuicios con homosexuales, que se vinculan al manejo de la visibilidad homosexual, que ocurre en las prácticas cotidianas en que las mujeres "se relacionan con estructuras de poder” (Meinerz, 2005: 128), y que se refieren tanto a la "administración del Estado, a través, por ejemplo, de las políticas de salud” (p. 128), como a los demás niveles en que se dan las relaciones en sociedad. Sobre eso, Touraine (2007), con base en interpretaciones de informaciones obtenidas con mujeres, afirma que "ser lesbiana (...) es una experiencia personal marcada por juicios sociales generalmente desfavorables, como todas las conductas que separan al placer sexual de la reproducción” (p. 38). Dar visibilidad o no a la homosexualidad remite a las maneras en que esas mujeres viven los prejuicios que están impregnados en determinados contextos de la sociedad, de los cuales, muchas veces, son víctimas. En el caso particular del grupo estudiado, nos dimos cuenta de que la forma de manejar la visibilidad de la homosexualidad se establece a través de las feminidades.

Es importante que comprendamos que al hablar en feminidades estamos considerando que el género es un concepto plural que no existe $a$ priori, sino que es una construcción, un proceso. Louro (1997: 22) considera que los géneros poseen “una fuerte invocación relacional”, es decir, los análisis sociales, a partir del concepto en cuestión, deben considerar tanto las masculinidades como las feminidades. Meyer (2005) amplía las posibilidades de articulaciones del concepto de género, afirmando que existen diversas construcciones sociales que, además de poseer representaciones de femenino y masculino, también producen y resignifican esas representaciones. Esas contribuciones no restringen el concepto género a la masculinidad y la feminidad hegemónica de una sociedad. En suma, adoptar el concepto género como categoría de análisis significa abarcar diferentes formas de masculinidad y feminidad.

Así, cuando decimos que en el grupo investigado las feminidades forman parte de las estrategias de manejo de la visibilidad de la homosexualidad, estamos considerando que es a través del proceso de construc- 
ción del ser mujer (que está relacionado con el ser hombre) que las jugadoras demarcan, tanto en el plano individual como en el colectivo, una postura frente al prejuicio. Encontramos en el equipo varias maneras de vivenciar la feminidad, desde la feminidad hegemónica de la sociedad hasta feminidades que se alejan de ella. Sin embargo, cuando la manera de portarse de una de las participantes se aproximaba a la masculinidad anhelada por la sociedad ${ }^{14}$, las jugadoras investigadas parecían despreciarla: "cuando la mujer es muy masculina, no sólo adopta el lado visual, ella quiere adoptar gestos, quiere adoptar hasta una cierta agresividad que es innecesaria” (Laura, 30/10/2007). Como se puede ver, Laura considera que las mujeres que poseen comportamientos cercanos a la masculinidad hegemónica no son bien vistas por ella. Esa postura pareció ser recurrente entre las jugadoras del Equipo, y está presente en las palabras de Denise, que destaca que eso está vinculado tanto en términos estéticos como actitudinales:

Creo que si tú eres mujer, tienes que vestirte como tal. Tú puedes tener tu opción, pero no necesitas transformarte en hombre (...). Mujer vistiéndose como hombre, vistiéndose y portándose [como hombre](...). Me parece feo, me parece vulgar, me parece asqueroso y creo que si es asqueroso para nosotros que somos homosexuales, imagínate para las personas que ya tienen un prejuicio. (Denise, 21/ 10/2007).

Ese consenso en el grupo investigado se asemeja al que Meinerz identificó en una investigación con mujeres homosexuales de clase media, donde encontró "una depreciación de la mujer masculinizada, calificada como camionera, que usa ropas y adopta comportamientos típicamente relacionados al género masculino" (2005: 84). El término nativo “camionera” también surgió en el estudio realizado por Dornelles (2004), con practicantes de fútbol de calle de Porto Alegre. Según parece, el término es compartido en el universo del fútbol femenino en Porto Alegre y es atribuido a las "mujeres (...) que participan del fútbol femenino, pero que también son homosexuales y presentan una construcción corporal y gestual que se acerca a las características que fueron construidas social e históricamente como masculinas” (Dornelles, 2004: 32).

Según la opinión de la mayoría de las jugadoras homosexuales del grupo, las personas son inducidas a caracterizar al homosexual por esos tipos de comportamientos. Entonces, el hecho de haber mujeres que posean trazos corporales y gestos masculinos hace con que el futsal sea visto como un universo de mujeres homosexuales; además, esas características refuerzan la vinculación de ese deporte con un universo de los hombres. Esa es una de las razones que lleva a las jugadoras del Equipo a considerar que la masculinización de las practicantes es dañina para el universo del fútbol

\footnotetext{
${ }^{14}$ En innumerables instituciones sociales es posible identificar características que son recomendadas para que los niños se hagan hombres, por ejemplo: virilidad, fuerza y agresividad.
} 
femenino brasileño; para ellas, es necesario eliminar esa etiqueta existente en el fútbol femenino. En otras palabras, es necesario deshacer la asociación entre fútbol femenino-masculinización-homosexualidad: "nosotras no tenemos que ponerle una etiqueta al fútbol femenino. Lo que tenemos que hacer es sacarle esa etiqueta” (Luana, 25/9/2007).

Así, al practicar el fútbol las jugadoras del grupo estudiado no siguen estrictamente la expectativa de la sociedad frente a las mujeres, que deben ser "femeninas, es decir, sonrientes, simpáticas, preocupadas, sumisas, discretas, recatadas o, incluso, apagadas" (Bourdieu, 2003: 83). Sin embargo, pese a ello no dejan de ser vanidosas, puesto que tienen cuidado con las ropas, las uñas y el pelo, acercándose a la delicadeza y vanidad de lo femenino hegemónico de la sociedad.

Es posible percatarse de eso cuando Graciele, al hablar sobre las vestimentas que utiliza para entrenar, afirma que "si yo estoy en la cuadra, algo está combinando allí. Algo tiene que combinar, de lo contrario no juego" (Graciele, 17/9/2007). A su vez, las practicantes Valéria y Denise se pintan las uñas demostrando fuertes preocupaciones estéticas culturalmente asociadas a las mujeres: en todos los momentos en que fueron observadas, estaban con las uñas pintadas con dibujos bastante delicados y con un color rosado, un color culturalmente femenino. La misma actitud se percibe en Ana, que usa zapatillas blancas con detalles en rosado y tiene, tatuada en el tobillo, una pelota de fútbol del mismo color, rodeada por flores.

Sin embargo, cuando entrenan o compiten esa vanidad femenina muchas veces es vivenciada junto con gestos viriles masculinos. Así, si los comportamientos femeninos son evidentes en las vestimentas y fuera del juego, durante los partidos las jugadoras actúan dentro de estándares normalmente asociados a los hombres. Con mucha frecuencia dividen la pelota ${ }^{15}$ utilizando el cuerpo de manera viril e, incluso, violenta. Usualmente, después de los juegos, las practicantes estudiadas tienen marcas en el cuerpo provocadas por las jugadas más duras o agresivas, situaciones en que hematomas, hinchazones, arañazos y otras lesiones son comunes en sus cuerpos. Sin embargo, preocupadas en mantener un cuerpo dentro de estándares femeninos, nuestras colaboradoras siempre trataron de esconder las marcas que traían de sus encuentros deportivos. Un ejemplo fue la situación en que Glória utilizó maquillaje para cubrir un hematoma en la pierna; otro ejemplo, el hecho de que Valéria (que es la arquera del Equipo) no quiso dejar que le sacaran fotos a sus uñas (pintadas) después del juego, justificando que, en ese momento, sus dedos estaban "feos" por las hinchazones que tenía debido a la práctica del fútbol. Ese comportamiento es muy distinto a lo que ocurre con los hombres, en deportes (incluso en el fútbol) donde marcas corporales como esas son vistas como símbolos que les dan status en sus respectivos contextos.

\footnotetext{
${ }^{15}$ Jerga del fútbol que, en Brasil, significa disputar el dominio de la pelota de manera intensa, vigorosa, agresiva e incluso violenta.
} 


\section{El futsal femenino vivido como un espacio de ocio para mujeres homosexuales}

Los espacios de ocio destinados a mujeres homosexuales son escasos en la ciudad de Porto Alegre. Meinerz (2005: 66) dedicó un capítulo de su disertación a hablar sobre "los espacios de la colaboración homoerótica femenina”. Los hallazgos de esa autora parecen aproximarse a aquellos identificados en las entrevistas que realizamos, en las cuales se encuentra la misma queja. Meinerz cuenta que escuchó “diversas quejas de las mujeres sobre la inexistencia de un lugar gay para mujeres en Porto Alegre”16 (Ibid: 82). En palabras de Laura, nuestra colaboradora, "los espacios son pocos [y] no son muy variados. Entonces yo creo que falta, así, un poco (...) de lugares”.

De la misma forma que Laura, las mujeres investigadas por Meinerz, además de quejarse de los pocos lugares de ocio existentes para el público de lesbianas, también los descalifican. En palabras de Cíntia, una de las investigadas por la autora, es posible observar argumentos que descalifican uno de los únicos lugares específicos para mujeres homosexuales en Porto Alegre:

Es por el público que va allá, sabe, porque hay muchas peleas y también porque no se puede querer ir con las músicas que ellos tocan. Nosotros fuimos el año nuevo, porque hay una fiesta buena y no hay nada en otra parte, pero ni con eso nos pudimos quedar mucho rato. (Pregunto por qué) ¿ Tú te ibas a poder quedar en un lugar que sólo toca Bonde do Tigrão y Égüinha Pocotó? ¡Por favor! (Ibid: 83).

El estudio de Meinerz se centró en lugares de ocio que no son específicos para lesbianas, pues sus investigadas, mujeres homosexuales de clase media, no eran asiduas de los lugares de ocio vinculados a ese público en particular. La autora denominó esa elección de sus investigadas como una búsqueda por "sociabilidad fuera del gueto" (Ibid: 67). En el sentido utilizado por Meinerz, la expresión gueto, utilizada usualmente en el campo de estudio de la sociología para referirse a "redes de barrios habitados por negros, judíos o grupos provenientes de otra nacionalidad u origen étnico" (Ibid: 67), pasó a ser asociada a la homosexualidad para caracterizar lugares en que "se está entre iguales”, en "un lugar de protección y, al mismo tiempo, de exclusión” (Ibid).

En esa dirección están las afirmaciones de nuestras colaboradoras, esas que consideran que los encuentros proporcionados por el Equipo son momentos de ocio en sus cotidianos, donde ellas tienen la oportunidad de estar entre sus pares. En palabras de Julia, jugar al fútbol no significa sólo practicar el deporte en sí, "sino que es la convivencia con las personas". Queda claro, entonces, que la elección de participar, los fines de semana,

${ }^{16}$ Destado de la autora. 
en los entrenamientos y juegos oficiales es hecha por las practicantes también por la afinidad que hay entre ellas.

En el caso de la mayoría de las practicantes de futsal investigadas, observamos que no todos los espacios de ocio logran dejarlas cómodas para vivenciar el placer y estar relajadas, como se esperaría para ese momento de sus vidas. Por esa razón, los momentos que deberían ser vividos "por su propio placer, por su propia satisfacción (...) dentro de ciertos limites socialmente establecidos” (Elias y Dunning, 1992: 140) terminan siendo perjudicados por la no aceptación social de su condición de homosexuales. Ese aspecto se articula con la necesidad -ya mencionada-de manejo de la visibilidad que ellas dan a su opción sexual. Estar en un espacio público y vivir un momento de ocio con la novia, en que hay intercambio de cariños, favorece la discriminación y el prejuicio de transeúntes que -no raramente- comparten los ideales de "sectores [sociales] tradicionales" (Louro, 2004: 28). Es de esa forma que los encuentros proporcionados por el Equipo terminan siendo favorables para vivir sus momentos de ocio, puesto que posibilitan que las practicantes homosexuales no se expongan a los prejuicios.

En el estudio de Meinerz, el compartir una opción homosexual también proporciona un elemento de afinidad entre sus investigadas, permitiendo que éstas formen una red de relaciones sociales. Según afirma,

el hecho de compartir determinadas experiencias sexuales y afectivas, consideradas como socialmente desviadas en lo que se refiere a las expectativas de coherencia entre género y orientación erótica, constituye un importante elemento de afinidad que organiza las redes de relaciones entre las mujeres investigadas (Meinerz, 2005: 40).

Sin embargo, es importante destacar que "las redes [de mujeres homosexuales] investigadas no pueden ser definidas exclusivamente a partir de eso” (Ibid: 41). En el ámbito de las practicantes de futsal investigadas, es interesante destacar que el asociativismo de esas mujeres tampoco está pautado sólo por la cuestión de la elección sexual, sino también por el gusto de practicar futsal y por la amistad compartida ${ }^{17}$. Si aquí abordamos particularidades de las practicantes homosexuales, es por ser este uno de los sustentáculos del asociativismo estudiado, pero no es el único.

Importa, entonces, destacar que, a pesar de que no podemos incorporar el mundo del futsal investigado entre los guetos gays ${ }^{18}$, los encuentros proporcionados por el Equipo, e incluso entre los diferentes equipos que participan de ese contexto, pueden ser vistos como momentos en que

\footnotetext{
${ }^{17}$ En el texto original el tema de la amistad fue discutido, sin embargo, debido a los límites de ese trabajo, no lo desarrollaremos.

${ }^{18}$ En ese universo, ni el Equipo estudiado, ni los demás grupos participantes, son compuestos sólo por mujeres homosexuales.
} 
se aceptan y se respetan las diferentes formas de orientación sexual y, por lo tanto, son espacios "culturalmente delimitados, en los cuales la diferencia de orientación sexual se acepta y se respeta” (Ibid: 42). En esos espacios, la afinidad entre las practicantes del Equipo ocurre, entre otros aspectos, por la opción sexual que ellas tienen en común, lo que favorece que los encuentros proporcionados tengan significados cercanos a lo que Elias y Dunning consideran ocio. Para los autores, los momentos de ocio

representan una esfera de vida que ofrece más oportunidades a las personas para que experimenten un agradable estímulo de las emociones, una divertida excitación que puede ser experimentada en público, compartida con otros y disfrutada con aprobación social y buena consciencia (1992: 151).

Si, para vivir un momento de ocio, se anhela que exista el compartir y la aceptación social, la investigación nos mostró que para mujeres homosexuales no es en cualquier lugar que eso puede darse. Sobre ese aspecto, tanto nuestras colaboradoras como las de Meinerz afirman que Porto Alegre es una ciudad con poquísimos espacios de ocio para el público de lesbianas.

Pero cabe señalar que, aunque nuestras colaboradoras se muestren insatisfechas con la forma en que son tratadas por la sociedad en general, en ese contexto en que el prejuicio en relación a la homosexualidad femenina es casi inexistente el género funciona como un medio de distinción, donde el Equipo parece considerar positivo que no hayan mujeres “masculinizadas” o “camioneras” entre sus componentes. Así, aunque en ese universo el género se vuelve, también, un instrumento de jerarquización, pues allí hay innumerables tipos de feminidad que son respetados, es evidente que habría situaciones tensas para alguna jugadora que adoptara una feminidad muy próxima a las características que fueron social e históricamente construidas como masculinas.

Teniendo en vista el hecho de que practican el deporte en un contexto donde la homosexualidad se acepta, puede, además, decirse que el universo del grupo investigado es por nosotros interpretado como un espacio en que el manejo de la visibilidad de la homosexualidad es menos presente que en otros locales. Eso se asemeja a otros espacios de ocio que se constituyen como espacios homosexuales, como algunos bares y discotecas que existen en varias ciudades.

\section{Consideraciones finales}

Teniendo como base elementos históricos y culturales, asociados a hechos observados en el cotidiano y en la producción académica sobre los deportes, podemos afirmar que, en Brasil, el fútbol es el deporte que más llama la atención. Entre innumerables características que son usualmente vinculadas a él y que hacen con que Brasil sea considerado como el país del 
fútbol, destacamos que esa práctica social es fundamentalmente protagonizada por los hombres, siendo, así, considerada una actividad masculina. Eso no significa que mujeres estén ausentes del universo futbolístico, sino que, en este país, la presencia de ellas en el fútbol todavía causa alguna extrañeza, lo que se hace más evidente cuando ellas están efectivamente practicando ese deporte.

Esta extrañeza nos llevó a tratar de comprender el asociativismo deportivo de un grupo de mujeres practicantes de fútbol, a partir de un estudio etnográfico en la ciudad de Porto Alegre (Brasil). El análisis desde adentro del universo estudiado nos permitió comprender, desde el punto de vista nativo, los significados del fútbol para aquellas mujeres y las dinámicas de desarrollo de sus actividades. Después de un año de observaciones encontramos un conjunto de elementos que nos llevaron a reflexionar sobre las formas de apropiación del fútbol en ese contexto, así como las relaciones que se establecían entre esa práctica y el hecho de que varias de las participantes del Equipo son homosexuales.

A medida que transcurrían las observaciones, nos percatamos de que el asociativismo deportivo vivido por las mujeres estudiadas se pautaba en innumerables formas de interacciones sociales, donde se evidenciaban vínculos de parentesco, de relaciones profesionales, de afinidades deportivas y lazos relacionados a la sexualidad. Esas conexiones establecían las lógicas de funcionamiento del grupo, donde se articulaban, jerárquicamente, expectativas acerca de maneras de ser del Equipo y de cada individuo en particular. Aunque no todas las participantes compartían todos esos vínculos, eso no era impedimento para que se mantuviesen juntas en ese contexto, donde la práctica del deporte era el mayor lazo que sustentaba la existencia del grupo.

En lo que se refiere al deporte, la dinámica del equipo se materializa en entrenamientos, partidos amistosos y partidos en competiciones. Al mismo tiempo en que las investigadas afirman jugar por diversión, destacan la importancia de asumir compromisos con el grupo, la valorización del capital deportivo de cada una, la relevancia de las ganas de vencer y de buscar buenos resultados deportivos. Así, para ser una jugadora del equipo es necesario demostrar un saber hacer que se vincula a tener conocimientos y dominios prácticos de aspectos técnico-tácticos del futsal, así como disputar, partido a partido, un lugar en el equipo principal. Pero eso no es suficiente: en ese contexto también es necesario tener una buena convivencia con las compañeras y compartir juegos, momentos de relajación que ocurren en los entrenamientos y en actividades que el equipo realiza fuera de la cuadra. De acuerdo con nuestras observaciones y con testimonios de las jugadoras, la manera de vivenciar el deporte oscila entre la seriedad y la diversión, la búsqueda de un buen rendimiento y el jugar por placer, aspectos que terminan caracterizando un ethos peculiar del grupo estudiado.

En lo que se refiere a la homosexualidad, constatamos que ésta se entrelaza con algunas dimensiones del universo deportivo investigado, así 
como con otros elementos que ocurren fuera de él. Tanto en el contexto del fútbol como en su exterior, las jugadoras manejan la visibilidad de la homosexualidad haciendo uso de estrategias articuladas con la feminidad hegemónica. Específicamente en relación con el deporte que escogieron practicar, preocupadas en no fortalecer una asociación entre fútbol femenino, masculinización y homosexualidad, critican a las jugadoras que se caracterizan como "camioneras". Eso ocurre, pues en las acciones y en los discursos de las jugadoras investigadas fue posible observar que ellas no identifican su sexualidad como la esfera más importante de sus vidas; luego, creen que no necesitan darle visibilidad, pues también están de acuerdo en que hay muchos prejuicios en la sociedad con respecto a ese tema.

Nos dimos cuenta, también, de que en los espacios donde fue realizada la investigación el manejo de la visibilidad de la homosexualidad es minimizado, pues ahí el prejuicio relativo a la sexualidad es casi imperceptible. De esa forma, los lugares donde ellas viven el fútbol terminan constituyéndose en espacios privilegiados para convivir en sus momentos de ocio. 


\section{Bibliografía}

Becker, Howard S. (1997), Métodos de Pesquisa em Ciências Sociais. $3^{\mathrm{a}}$ ed., São Paulo, ed. Hucitec, Brasil.

Betti, Mauro (2004), Violência em campo: dinheiro, mídia e transgressão às regras no futebol espetáculo. Ijuí, Editora Unijuí, Brasil.

Bourdieu, Pierre (2003), A dominação masculina. $3^{\circ}$ edición, Rio de Janeiro, ed. Bertrand Brasil, Brasil.

Costa, Leda Maria (Nov2006/Feb2007), “O que é uma torcedora? Notas sobre a representação e auto-representação do público feminino de futebol”. In: Esporte e Sociedade, Ano 2, número 4, Nov2006/Feb2007. Site http:// www.lazer.eefd.ufrj.br/espsoc/ Accesado en 17/03/2006.

Da Matta, Roberto (1987), Relativizando - uma introdução à Antropologia Social. Petrópolis, ed. Vozes, Brasil.

Damo, Arlei Sander (2007), “A rua e o futebol”. In: Stigger, Marco Paulo; González, Fernando; e Silveira, Raquel da. O esporte na cidade: estudos etnográficos sobre sociabilidades esportivas em espaços urbanos. Porto Alegre, Editora de la UFRGS, Brasil.

Idem (2006), “O ethos capitalista e o espírito das copas”. In: Gastaldo, Édison Luis e Guedes, Simoni Lahud (organizadores). Nações em Campo: Copa do mundo e identidade nacional. Niterói, ed. Intertexto, Brasil.

Idem (2003), "Monopólio estético e diversidade configuracional no futebol”. In: Movimento. Porto Alegre, Brasil.

Idem (2002), Futebol e identidade social: uma leitura antropológica das rivalidades entre torcedores e clubes. Porto Alegre, Editora Universidade, Brasil.

Daólio, Jocimar (2005), “A superstição no futebol brasileiro”. In: Daólio, Jocimar (Org.). Futebol, cultura e sociedade. Campinas, ed. Autores Associados, Brasil.

Dornelles, Priscila Gomes (2004). O futebol feminino de várzea: uma análise cultural. Monografia da Especialização Pedagogias do Corpo e da Saúde Escola de Educação Física, Universidade Federal do Rio Grande do Sul, Porto Alegre, Brasil

Dumazedier, Jofre (1973), Lazer e cultura popular. São Paulo, Editora Perspectiva S. A, Brasil.

Elias, Nobert; Dunning, Eric (1992), “O lazer no espectro do tempo livre”. In: Elias, Nobert e Dunning, Eric. A busca da excitação. Lisboa: Difusão Editorial, Lda, Brasil. 
Foucault, Michel (2006), História da sexualidade I: a vontade de saber. $17^{\circ}$ edición, Rio de Janeiro: Edições Graal, Brasil.

Geertz, Clifford (1989), A interpretação das culturas. Rio de Janeiro, ed. Guanabara e Koogan, Brasil.

Guedes, Simoni Lahud (2006), “De criollos e capoeiras: notas sobre futebol e identidade nacional na Argentina e no Brasil”. In: Gastaldo, Édison Luis e Guedes, Simoni Lahud (organizadores). Nações em Campo: Copa do mundo e identidade nacional. Niterói, ed. Intertexto, Brasil.

Howe, Davi P. (2001), “An ethnography of pain and injury in Professional Rugby Union”. In: International Review for the Sociology of Sport, UK.

Knoppers, Annelies; Anthonissen, Anton (2003), "Women's soccer in the United States and the Netherlands: differences and similarities in regimes of inequalities”. In: Sociology of Sports Journal, Canada.

Laplantine, François (1994), Aprender Antropologia. $8^{a}$ ed. São Paulo, ed. Brasiliense, Brasil.

Louro, Guacira Lopes (2004), Um corpo estranho: ensaios sobre sexualidade e teoria queer. Belo Horizonte, Editora Autêntica, Brasil.

Idem (1997). Gênero, sexualidade e educação: uma perspectiva pósestruturalista. $8^{\mathrm{a}}$ edición, Petrópolis, Editora Vozes, Brasil.

Magnani, José Guilherme Cantor (1984), Festa no pedaço: cultura popular e lazer na cidade. São Paulo, ed. Brasiliense S. A., Brasil.

Marivoet, Salomé (1998), Aspectos sociológicos do desporto. Lisboa, Livros Horizontes, Portugal.

Meinerz, Nádia Elisa (2005), Entre mulheres: estudo etnográfico sobre a constituição da parceria homoerótica feminima em segmentos médios na cidade de Porto Alegre - RS. Dissertação (mestrado) - Universidade Federal do Rio Grande do Sul. Instituto de Filosofia e Ciências Humanas. Programa de Pos Graduación en Antropología social, Porto Alegre, Brasil.

Mennesson, Christine (2005), Etre une femme dans le monde des hommes: socialisation sportive er construction du genre. L'Harmattan, France.

Idem (2004), "Les processus de construction et de modification des dispositions sexuées des femmes investies dans um sport dit 'masculin' ”. In : Societe de Sociologie du Sport de Langue Française. Dispositions et pratiques sportives: débats actuels en sociologie du sport., L'Hamattan, France 2004.

Mennesson, Christine and Clément, Jean-Paul (2003), “Homosociability 
and homosexuality: the case of soccer played by women”. In: International Review for the Sociology of Sport, Canada.

Meyer, Dagmar Estemann (2005), “Gênero e educação: teoria e política”. In: Louro, Guacira Lopes; Felipe, Jane; Goellner, Silvana Vilodre (organizadoras). Corpo, gênero e sexualidade: um debate contemporâneo na educação. $2^{\circ}$ edición, Petrópolis, ed. Vozes, Brasil.

Moura, E. L. (2005), “O futebol como área reservada masculina”. In: Daolio, J. (Org.). Futebol, cultura e sociedade. Campinas, ed. Autores Associados, Brasil.

Oliveira, Roberto Cardoso de (1998). “O trabalho do Antropólogo: olhar, ouvir, escrever”. In: Oliveira, Roberto Cardoso de, O Trabalho do Antropólogo. Brasília/ São Paulo, ed. Paralelo Quinze/Editora de la Unesp, Brasil.

Pfister, Gertrud (mayo/agosto de 2003), "Líderes femininas em organizações esportivas - tendências mundiais”. In: Revista Movimento. Porto Alegre, Brasil.

Ruquoy, Danielle (1997), "Situação de entrevista e estratégia do entrevistador”. In: Albarello, Luc; Digneffe, Françoise; Hiernaux, Christian M., et al. Práticas e Métodos de Investigação em Ciências Sociais. Lisboa: Gradiva, Brasil.

Rybezynski, Witold (1991), "Waiting for the Weekend”. The Athlantic Mounthly. v.268, n.2, p.35-52.

Idem (2000). Esperando o fim de semana. Rio de Janeiro/São Paulo, ed. Record Editora, Brasil.

Simmel, Georg (1983). Sociologia. Organizador (da coletânea) Evaristo de Moraes Filho, São Paulo, ed. Ática, Brasil.

Soares, Antônio Jorge; Lovisolo, Hugo, (2001), “O futebol é fogo de palha: a profecia de Graciliano Ramos”. In: Helal, Ronaldo; Soares, Antônio Jorge; Lovisolo, Hugo (organizadores). A invenção do país do futebol: mídia, raça e idolatria. Rio de Janeiro, ed. Mauad, Brasil.

Soares, Antônio Jorge G (1994), Futebol, malandragem e identidade. Vitória, ed. SPDC/UFES, Brasil.

Stigger, Marco Paulo (2007), “Estudos etnográficos sobre esporte e lazer: pressupostos teórico-metodológicos e pesquisa de campo”. In: Stigger, Marco Paulo; González, Fernando; e Silveira, Raquel da. O esporte na cidade: estudos etnográficos sobre sociabilidades esportivas em espaços urbanos. Porto Alegre: Editora de la UFRGS, p. 31 - 50. 
Idem (1997), “Futebol de veteranos: um estudo etnográfico sobre o esporte no cotidiano urbano”. In: Revista Movimento, Brasil.

Thomassim, Luís Eduardo (2007), “Imagens das crianças da periferia em projetos sociais esportivos”. In: Stigger, Marco Paulo; González, Fernando; e Silveira, Raquel da. O esporte na cidade: estudos etnográficos sobre sociabilidades esportivas em espaços urbanos. Porto Alegre: Editora de la UFRGS, Brasil.

Toledo, Luiz Henrique (2002), Lógicas no futebol. São Paulo: Editora Hucitec, Brasil.

Touraine, Alain (2007). O mundo das mulheres. Petrópolis, ed. Vozes, Brasil.

Winkin, Yves (1998), “Descer ao campo”. In: Winkin, Yves A nova comunicação: da teoria ao trabalho de campo. Campinas, ed. Papirus, Brasil. 